\title{
A Pharmacological Batch of Mongersen that Downregulates Smad7 is Effective as Induction Therapy in Active Crohn's Disease: A Phase II, Open-Label Study
}

\author{
Irene Marafini ${ }^{1,2} \cdot$ Carmine Stolfi $^{1} \cdot$ Edoardo Troncone $^{1,2} \cdot$ Elisabetta Lolli $^{2} \cdot$ Sara Onali ${ }^{3,4}$. \\ Omero Alessandro Paoluzi ${ }^{1,2} \cdot$ Massimo C. Fantini ${ }^{3,4} \cdot$ Livia Biancone $^{1,2} \cdot$ Emma Calabrese $^{1,2} \cdot$ Antonio Di Grazia $^{1}$. \\ Ivan Monteleone ${ }^{1} \cdot$ Marco Vincenzo Lenti $^{5}$ - Antonio Di Sabatino ${ }^{5}$. Giovanni Monteleone ${ }^{1,2}$
}

Accepted: 29 March 2021 / Published online: 19 April 2021

(c) The Author(s) 2021

\begin{abstract}
Background A recent phase III trial did not confirm the previous clinical and endoscopic improvements seen in patients with Crohn's disease (CD) receiving Mongersen, an oral Smad7 antisense oligonucleotide. Factors accounting for such a discrepancy are unknown.

Objective Our objective was to further assess whether Mongersen was effective as induction therapy in active CD and evaluate the in vitro inhibitory effect of various batches of Mongersen used in the previous and present trials on Smad7 expression. Methods In a phase II, open-label study, 18 patients with active CD (Crohn's Disease Activity Index [CDAI] score $>220$ and evidence of endoscopic lesions) received Mongersen $160 \mathrm{mg} /$ day for 12 weeks. The rates of clinical remission, defined as $\mathrm{CDAI}<150$, and clinical response, defined as a CDAI score decrease $\geq 100$, were evaluated at week 4,8 , and 12 . The fraction of circulating CCR9-expressing leukocytes was assessed by flow cytometry. Smad7 expression was evaluated in the human colorectal cancer cell line HCT-116 transfected with different batches of Mongersen using real-time polymerase chain reaction (PCR) and Western blotting,

Results The proportions of patients experiencing clinical remission were $38.9 \%, 55.6 \%$, and $50.0 \%$ at week 4, 8, and 12 , respectively. At the same time points, the rates of clinical response were $72.2 \%, 77.8 \%$, and $77.8 \%$, respectively. Mongersen reduced the percentages of CCR9-expressing CD45+ cells. The batch of Mongersen used in this study, but not two batches used in the phase III study, inhibited Smad7 expression in HCT-116 cells.

Conclusions The present findings support the clinical benefit of Mongersen in active CD and show that various batches manufactured during the GED0301 program differ in their ability to inhibit in vitro Smad7.
\end{abstract}

Trial Registration Number NCT02685683; EudraCT 2015-001693-18

\section{Key Points}

Irene Marafini, Carmine Stolfi have contributed equally.

Giovanni Monteleone

Gi.Monteleone@Med.uniroma2.it

1 Dipartimento di Medicina dei Sistemi, Università di Roma "Tor Vergata", Via Montpellier, 1, 00133 Rome, Italy

2 Gastroenterology Unit, Fondazione Policlinico Tor Vergata, Rome, Italy

3 Gastroenterology Unit, Duilio Casula Hospital, AOU Cagliari, Cagliari, Italy

4 Department of Medical Science and Public Health, University of Cagliari, Cagliari, Italy

5 Department of Internal Medicine, San Matteo Hospital Foundation, University of Pavia, Pavia, Italy
This was a small open-label study showing clinical benefit in patients with active Crohn's disease receiving Mongersen, a Smad7 antisense oligonucleotide-containing oral compound.

The study confirms the good safety profile of Mongersen.

Various batches of Mongersen developed and manufactured for the clinical trials differ in their ability to downregulate Smad7 expression in cultured cells. 


\section{Introduction}

Crohn's disease (CD) is a chronic, disabling, relapsing inflammatory disease that mainly affects the terminal ileum and right colon, although it may involve any segment of the gastrointestinal tract [1]. The etiology of CD is unknown, but it is considered to be an immune-mediated disease in which a combination of genetic, environmental, and immunologic factors contribute to trigger a pathologic process leading to the tissue damage [2].

Patients with CD may present with symptoms that include abdominal pain, diarrhea, and weight loss, and the course of the disease can be associated with systemic symptoms such as malaise, anorexia, or fever [3]. Common complications are intestinal strictures and fistulas, with an increased frequency over time, occurring in more than half of the patients by 20 years after diagnosis, and often requiring surgery [4].

Despite significant advancements in the understanding of the basic mechanisms underlying the tissue-damaging inflammatory response, treatment of patients with CD remains a difficult challenge [5]. The natural history of $\mathrm{CD}$ is characterized by a remitting and relapsing course that progresses to complications and surgery in the majority of patients [4]. A stepwise approach according to disease location and severity at presentation has been advocated, with the primary aim of inducing and maintaining clinical remission, improving quality of life, and minimizing shortterm and long-term toxicity and complications $[6,7]$. Treatment of CD currently involves pharmacological treatment and surgery, the latter of which is indicated for medically refractory disease and/or complications $[8,9]$. Pharmacological treatment includes traditional anti-inflammatory drugs, immunomodulators, and biologic compounds, such as tumor necrosis factor (TNF)- $\alpha$ blockers, interleukin (IL)-12/ IL-23 blockers, and integrin antagonists [6]. However, not all patients respond to these drugs, and therapy can be associated with significant potential adverse events [10]. There is a clear need for newer therapeutic approaches in patients with $\mathrm{CD}$ who do not respond, lose response, or are intolerant to currently available treatments, as well as to improve the toxicity associated with chronic immunosuppression observed with corticosteroids.

Accumulating evidence indicates that the CD-associated chronic inflammation is caused by an excessive local immune response to luminal antigens that is not appropriately controlled by the normal counterregulatory mechanisms [2]. One of the counterregulatory mechanisms involves transforming growth factor (TGF)- $\beta 1$, which is a multifunctional factor that has been shown to play an important role in the control of immune homeostasis and to act as a potent negative regulator of mucosal inflammation [11-13]. In CD, activity of TGF $\beta 1$ is defective because of high levels of Smad7, an intracellular inhibitor of the TGF $\beta 1 /$ Smad signaling $[11,14]$. The functional relevance of these findings was supported by mechanistic studies showing that inhibition of Smad7 with a specific antisense oligonucleotide restored the TGF $\beta 1$-mediated suppression of mucosal inflammation in murine models of colitis [15, 16]. Moreover, phase I and II studies documented a clinical and endoscopic benefit in patients with $\mathrm{CD}$ treated with an oral compound, named Mongersen (previously GED0301), containing the Smad7 antisense oligonucleotide [12, 17, 18]. However, a recent phase III trial was prematurely discontinued following a futility analysis showing no clinical and endoscopic benefit in patients with CD treated with Mongersen [19]. Surprisingly, in this study, the percentages of patients achieving clinical response or clinical remission following Mongersen treatment did not differ from those seen in placebo-treated patients and were markedly lower than those documented in the previous phase I and II studies. The reasons for these discrepancies remain unknown, although it has been hypothesized that they could, at least in part, rely on differences in the inclusion criteria between the phase II and III studies [20]. In fact, the phase II study did not require endoscopy for enrolment of patients, whereas the phase III study included patients with active ulcerations observed in the terminal ileum and/or colon on ileocolonoscopy. However, the inclusion criteria adopted in the phase III study were similar to those of the endoscopic open phase II study in which Mongersen was beneficial in patients with active $\mathrm{CD}$ even though no placebo group was included [21]. During the whole Mongersen project, many different batches of the drug substance have been manufactured and used in clinical trials. The identity of the investigational product and the stability of each formulation were checked during the clinical studies and after the end of the phase III study. However, it remains unclear whether physical and chemical changes of the batches, which can occur during large-scale synthesis of antisense oligonucleotides, have contributed to the differences in the results of the previous studies.

Other clinical studies were conducted in parallel with the phase III study, although the sponsor terminated these studies early after the cancellation of the GED0301 clinical program and the decision to terminate the phase III study. In particular, a phase II open-label study was aimed at further exploring the clinical and pharmacodynamic effects of Mongersen in patients with active $\mathrm{CD}$. This study used a different batch of Mongersen from those used in the phase III study.

We present the clinical data of this phase II study. Moreover, we assessed the in vitro pharmacological activity of various batches of Mongersen used in the clinical trials. 


\section{Methods}

\subsection{Study Design}

This was a phase II open-label study, designed without a placebo or active control, that focused on further understanding the clinical outcomes in patients with active $\mathrm{CD}$ treated with Mongersen $160 \mathrm{mg} /$ day for 12 weeks as compared with baseline. All subjects were assigned to receive the same treatment: four 40 -mg tablets daily during the 12-week induction period. The dose of $160 \mathrm{mg}$ was selected to provide subjects with the highest safe and effective treatment dose of Mongersen based on the clinical safety and efficacy data from the prior Mongersen studies [12, 17]. Patient eligibility was determined during a 4-week screening period, during which patient demographics and medical histories were obtained and the following information collected: Crohn's Disease Activity Index (CDAI), patient diary, viral serology, stool cultures, Simple Endoscopic Score for Crohn's Disease (SES-CD), fecal calprotectin, vital signs, and laboratory evaluations. The study was conducted at the Tor Vergata University Hospital (Rome, Italy) and Ospedale San Matteo (Pavia, Italy). The study was carried out in accordance with the Declaration of Helsinki, the good clinical practice guidelines established by the International Conference on Harmonisation, and the applicable drug and data protection laws and regulations existing in Italy. Protocols, amendments, and informed consent documentation were reviewed and approved by the institutional review boards and independent ethics committee of each study center before the trial began. Written informed consent was obtained from patients before they underwent screening for eligibility.

\subsection{Patients}

Eligible patients were aged $\geq 18$ years with a documented history of $\mathrm{CD}$ for $\geq 3$ months before screening, with inflammatory lesions in the terminal ileum, colon, or both. Patients had active CD defined by a CDAI score $\geq 220$ and $\leq 450$ and either a total SES-CD $\geq 6$ or ileum segmental SES-CD $\geq 4$ and had experienced failure or intolerance to at least one of the following: budesonide, systemic corticosteroids, immunosuppressants (e.g., azathioprine, 6-mercaptopurine, or methotrexate), or biologics. Ileocolonoscopy was performed during the screening period (baseline), and video images of all endoscopic procedures were captured and sent to a qualified centralized reader for calculation of the SES-CD.

Because the active compound of Mongersen is mainly released in the distal ileum and right colon, we excluded patients with lesions in the stomach and proximal small intestine. Patients were also excluded if they had a diagnosis of ulcerative colitis, indeterminate colitis, ischemic colitis, microscopic colitis, radiation colitis, or diverticular disease-associated colitis. Patients were excluded if they had abscesses, short bowel syndrome, or intestinal strictures with pre-stenotic dilatation, requiring procedural intervention or not passable with an adult colonoscope, or any CD complications for which surgery might have been indicated or could have confounded the evaluation of efficacy. We also excluded patients with an intestinal resection within 6 months or any intra-abdominal surgery within 3 months prior to screening visit, those who had an ileostomy or a colostomy, and patients who had received treatments with mycophenolic acid, tacrolimus, sirolimus, cyclosporine, thalidomide, or apheresis within 8 weeks prior to screening visit, or intravenous corticosteroids within 2 weeks prior to screening visit. Patients could continue to receive stable doses of oral mesalamine and/or prednisone ( $\leq 20 \mathrm{mg}$ per day) or budesonide ( $\leq 9 \mathrm{mg}$ per day) through the study period. Patients could also receive a stable dose of immunomodulators (e.g., azathioprine, 6-mercaptopurine, or methotrexate) if therapy had been initiated $\geq 12$ weeks before initiation of the study treatment. The dose of immunosuppressants was required to be stable for $\geq 8$ weeks before study initiation and to remain stable through the study period. Patients could not receive treatment with topical 5-aminosalicylic acid or corticosteroid enemas or suppositories within 2 weeks, antibiotics within 3 weeks, or bile acid sequestrant before the date of their enrolment in the trial and throughout the study duration. Prior treatment with investigational biologics for the treatment of CD or with more than two TNF $\alpha$ blockers was not allowed. Patients could not receive treatment with a TNF $\alpha$ blocker within 8 weeks prior to the screening visit and throughout the entire duration of the study or with parenteral nutrition within 4 weeks prior to the screening visit. Patients with an active or recent infection or a history of cancer were excluded. Female participants were required to use a highly effective form of contraception throughout the study. We excluded women who were pregnant or breastfeeding, people with a clinically significant abnormality on electrocardiography or laboratory testing, and patients who had received prior treatment with Mongersen or had participated in a clinical study involving Mongersen.

\subsection{Efficacy and Safety Assessment}

The efficacy endpoints included the proportion of subjects achieving clinical remission (defined as a CDAI score < 150 ) and clinical response (defined as a CDAI score decrease of $\geq 100$ points) at week 4,8 , and 12 , and the change from baseline in the two-item patient-reported outcome (PRO2) score at week 4,8 , and 12 . Additionally, the changes from baseline in fecal calprotectin and high-sensitivity serum C-reactive protein (hsCRP) at week 12 were also assessed. 
Ileocolonoscopy was performed during the screening period (baseline) and at week 12. Video images of all endoscopic procedures were captured and sent to a qualified centralized reader for calculation of the SES-CD. Another endpoint was the evaluation of safety and tolerability of Mongersen. For this purpose, clinical, biochemical, and hematologic variables were periodically assessed. An enzyme-linked immunosorbent assay was used to monitor the patients for complement activation (a side effect of systemic antisense exposure). The severity of adverse events and their cause (study drug or procedure) was determined.

\subsection{Flow Cytometry Analysis}

Peripheral blood samples were collected into heparinized tubes at baseline and at week 12 following Mongersen treatment. Peripheral blood mononuclear cells (PBMC) were obtained by density-gradient centrifugation, washed three times in cold phosphate-buffered saline (PBS) and used to assess cell surface markers by flow cytometry. Since PBMC were processed immediately at the Tor Vergata University, no sample was collected at the San Matteo Hospital. Samples were available from only 12 of 13 patients enrolled at the Tor Vergata University Hospital as one patient did not give consent to collect blood. PBMC were stained with the following antibodies: anti-CD45-APC-H7, anti-CD3-Pacific Blue, and anti-CCR9-APC (all from BD Bioscience, San Jose, CA, USA). All antibodies were used at 1:50 final dilution. Appropriate isotype-matched controls were included in all of the experiments. Cells were analyzed using a Beckman Coulter Gallios cytometer and Kaluza software.

\subsection{Cell Culture}

The human colorectal cancer (CRC) cell line HCT-116 was obtained from the American Type Culture Collection and maintained in McCoy's 5A (Lonza, Verviers, Belgium) supplemented with $10 \%$ fetal bovine serum (FBS) (Euroclone, Milan, Italy) and $1 \%$ penicillin/streptomycin (Lonza), in a $37^{\circ} \mathrm{C}, 5 \% \mathrm{CO}_{2}$, fully humidified incubator. The cell line has been recently authenticated by short tandem repeat (STR) DNA fingerprinting using the PowerPlex 18D System kit according to the manufacturer's instructions (Promega, Milan, Italy). The STR profile matched the known DNA fingerprint.

To perform transfection experiments, $1 \mathrm{~mL}$ of single-cell suspension $\left(2 \times 10^{5}\right.$ cells $\left./ \mathrm{mL}\right)$ was added per well in six-well culture dishes. The day after, cells were washed with PBS (Lonza) and transfected with different batches of Mongersen using Opti-MEM I transfection medium plus lipofectamine 3000 reagent (Thermo Fisher Scientific, Monza, Italy) according to the manufacturer's instructions. The transfection was performed in a final volume of $2 \mathrm{~mL}$ to get a $1 \mu \mathrm{g} /$
$\mathrm{mL}$ final concentration of each test item. Cells incubated with Opti-MEM I transfection medium and lipofectamine 3000 only were used as internal control. After $24 \mathrm{~h}$, cells were washed with PBS and cultured with McCoy's 5A supplemented with $10 \%$ FBS and antibiotics for a further $24 \mathrm{~h}$. Cells were then washed with PBS, and total proteins were extracted to perform western blotting experiments. To evaluate RNA expression, cells were transfected as indicated and collected after $3 \mathrm{~h}$.

\subsection{RNA Extraction, Complementary DNA Preparation, and Real-Time Polymerase Chain Reaction}

RNA was extracted using PureLink messenger RNA mini kit (Thermo Fisher Scientific), according to the manufacturer's instructions. A constant amount of RNA ( $1 \mu \mathrm{g} / \mathrm{sample})$ was reverse transcribed into complementary DNA (cDNA), and $1 \mu \mathrm{L}$ of cDNA/sample was then amplified using real-time PCR and iQ SYBR Green Supermix (Bio-Rad Laboratories, Milan, Italy) and the following $\beta$-actin primers: FWD: 5'-AAGATGACCCAGATCATGTTTGAGACC-3'; REV: 5'-AGCCAGTCCAGACGCAGGAT-3'. Smad7 RNA expression was evaluated using a Taqman assay (Life Technologies). Smad7 RNA expression was calculated relative to the housekeeping $\beta$-actin gene on the base of the $\Delta \Delta \mathrm{Ct}$ algorithm.

\subsection{Western Blotting}

Total proteins were extracted using the following lysis buffer: $10 \mathrm{mmol} / \mathrm{L}$ HEPES, $1 \mathrm{mmol} / \mathrm{L}$ EDTA, $60 \mathrm{mmol} / \mathrm{L}$ $\mathrm{KCl}, 0.2 \%$ Igepal CA-630, $1 \mathrm{mmol} / \mathrm{L}$ sodium fluoride, 10 $\mu \mathrm{g} / \mathrm{mL}$ aprotinin, $10 \mu \mathrm{g} / \mathrm{mL}$ leupeptin, $1 \mathrm{mmol} / \mathrm{L}$ DTT, and $1 \mathrm{mmol} / \mathrm{L}$ PMSF (all from Sigma-Aldrich, Milan, Italy), separated on a $10 \%$ SDS-PAGE gel, and blots were then incubated with a mouse antihuman monoclonal SMAD7 antibody $(0.5 \mu \mathrm{g} / \mathrm{mL}$ final dilution; R\&D systems, Minneapolis, MN, USA) followed by a rabbit antimouse antibody conjugated to horseradish peroxidase (1:20000 final dilution; Dako, Milan, Italy). After analysis of Smad7, each blot was stripped and incubated with a mouse-antihuman monoclonal $\beta$-actin antibody (Sigma-Aldrich) to confirm equal loading of the lanes. Computer-assisted scanning densitometry (Image Lab Software, Bio-Rad Laboratories) was used to analyze the intensity of the immunoreactive bands.

\subsection{Analysis and Quantification of Cell Proliferation}

Cell proliferation was assessed using carboxyfluorescein diacetate succinimidyl ester (CFSE; Molecular Probes, Eugene, OR, USA), which covalently binds cell components to yield a fluorescence that is divided equally between 
daughter cells at each division. Briefly, $1 \mathrm{~mL}$ of single-cell suspension $\left(2 \times 10^{5}\right.$ cells $\left./ \mathrm{mL}\right)$ was added per well in sixwell culture dishes. The next day, cells were washed with PBS and transfected with four different batches of Smad7 antisense oligonucleotides using Opti-MEM I transfection medium plus lipofectamine 3000 reagent according to the manufacturer's instructions. The transfection was performed in a final volume of $2 \mathrm{~mL}$ to get a $1 \mu \mathrm{g} / \mathrm{mL}$ final concentration of each test item. Cells incubated with Opti-MEM I transfection medium and lipofectamine 3000 only were used as internal control. After $24 \mathrm{~h}$, cells were washed with PBS and incubated with CFSE according to the manufacturer's instructions. After 30 minutes, cells were washed with PBS and cultured with McCoy's 5A supplemented with 10\% FBS and antibiotics for a further $24 \mathrm{~h}$. Finally, cells were collected, washed with PBS, and then incubated with $5 \mu \mathrm{g} /$ $\mathrm{mL}$ of propidium iodide (PI) for 15 minutes, at $4{ }^{\circ} \mathrm{C}$ in the dark. CFSE- and/or PI-positive cells were determined by flow cytometry, and the data were analyzed using ModFit LT 2.0 (Verity Software House, Inc., ME, USA).

\subsection{Statistical Analysis}

The study was exploratory only and was not powered. It had a planned enrolment of 20 subjects who were to complete 12 weeks of treatment. All efficacy analyses were conducted according to the intention-to-treat (ITT) principle and, therefore, the ITT population included all subjects who entered the study and received at least one dose of Mongersen. For assessments of change from baseline, a baseline value and at least one post-baseline value were also required. Treatment failure rules were applied to the efficacy analyses. Subjects who had initiated or increased the dose of concomitant medications that are known to be effective for the treatment of CD (corticosteroids, immunosuppressants, or biologics) were considered to be treatment failures from the date of the event onward. The proportion of subjects achieving clinical remission/clinical response was calculated by the Wilson score method with two-sided $95 \%$ confidence intervals (CIs). Sensitivity analyses were performed using "data as observed" and "last observation carried forward" methods.

Changes in fecal calprotectin and hsCRP and in the percentages of CCR9-expressing PBMC in subgroups of patients with clinical remission/response from baseline to week 12 were assessed using the Wilcoxon signed-rank test.

For the in vitro studies with HCT-116 cells, values derived from all the observations were expressed as mean \pm standard error of the mean (SEM) of at least three independent experiments. Data were analyzed using one-way analysis of variance followed by Dunnett's multiple comparison test. Significance was defined as $p$-values $<0.05$.

\section{Results}

\subsection{Demographic and Baseline Patient Characteristics}

Patients were screened to provide a planned minimum of 20 patients completing 12 weeks of treatment with Mongersen. The planned duration of the study was 2 years, but the sponsor terminated the study early. Of the 45 patients screened for enrolment, 27 were screen failures. The reasons for screen failure included the following: nine patients had a CDAI score $<220$ at screening, five patients had stool positivity for enteric pathogens, five patients did not have a total SES-CD $\geq 6$ or ileum segmental SES-CD $\geq 4$ at screening, four patients had CD-related intestinal strictures, two patients had a history of a clinically significant systemic disease, one patient had laboratory alterations, and one patient had prior treatment with more than two TNF $\alpha$ blockers. In total, 18 patients (13 enrolled at the Tor Vergata University Hospital and five enrolled at the San Matteo Hospital) were assigned to receive Mongersen $160 \mathrm{mg} /$ day for 12 weeks. Table 1 shows the demographic and baseline disease characteristics of the 18 patients. The median disease duration was 10.3 years (range $0.3-30.0$ ). The majority of patients had the disease for $\geq 5$ years, with four patients $(22.2 \%)$ having had it for $\geq 5$ to $<10$ years, and ten patients $(55.6 \%)$ having had it for $\geq 10$ years. Overall, $38.9 \%$ of patients had prior surgery for $\mathrm{CD}$ and $33.3 \%$ had prior exposure to $\mathrm{TNF} \alpha$ blockers. A total of $11.1 \%$ of patients reported baseline use of oral corticosteroids (oral prednisolone $15 \mathrm{mg} /$ day), and no patient reported baseline use of immunosuppressants. The mean \pm standard deviation baseline SES-CD (centrally read) score was $14.9 \pm 10.34$.

\subsection{Clinical Remission and Response}

Overall, the median baseline CDAI score was 288.8 (95\% CI 264.2-334). Out of 18 enrolled patients, 17 (94.4\%) completed the 12-week treatment and one discontinued the treatment after week 8 because of lack of efficacy.

Overall, of the 18 patients in the ITT population, seven $(38.9 \%)$, ten $(55.6 \%)$, and nine $(50.0 \%)$ achieved clinical remission at week 4,8 , and 12 , respectively (Fig. 1a). At these time points, $72.2 \%(n=13), 77.8 \%(n=14)$, and $77.8 \%(n=14)$ of patients in the ITT population achieved clinical response (Fig. 1b). For the ITT population, the median change from baseline in CDAI score was -140.5 $(95 \% \mathrm{CI}-165.9$ to -59.8$)$ at week $4(p=0.0007),-162.2$ (95\% CI -191.6 to -107.4$)$ at week $8(p<0.0001)$, and $-161.6(95 \% \mathrm{CI}-197.5$ to -73.4$)$ at week $12(p=$ 0.0019). Mean changes from baseline in CDAI score to each time point are shown in Fig. 2a. 
Table 1 Demographics and baseline characteristics (ITT population)

\begin{tabular}{|c|c|}
\hline $\begin{array}{l}\text { Demographic and baseline character- } \\
\text { istic }\end{array}$ & Mongersen $160 \mathrm{mg}(N=18)$ \\
\hline \multicolumn{2}{|l|}{ Age (years) } \\
\hline Median (range) & $42.0(18-56)$ \\
\hline \multicolumn{2}{|l|}{ Sex } \\
\hline Male & $11(61.1)$ \\
\hline Female & $7(38.9)$ \\
\hline \multicolumn{2}{|l|}{ Tobacco history } \\
\hline Never smoked & $10(55.6)$ \\
\hline Past smoker & $3(16.7)$ \\
\hline Current smoker & $5(27.8)$ \\
\hline \multicolumn{2}{|l|}{ Duration of CD (years) } \\
\hline Median (minimum; maximum) & $10.30(0.3 ; 30.0)$ \\
\hline$<2$ & $2(11.1)$ \\
\hline 2 to $<5$ & $2(11.1)$ \\
\hline 5 to $<10$ & $4(22.2)$ \\
\hline$\geq 10$ & $10(55.6)$ \\
\hline \multicolumn{2}{|l|}{ Prior procedures or surgeries for $\mathrm{CD}$} \\
\hline Yes & $7(38.9)$ \\
\hline No & $11(61.1)$ \\
\hline \multicolumn{2}{|l|}{ Previous exposure to $\mathrm{TNF} \alpha$ blockers } \\
\hline Yes & $6(33.3)$ \\
\hline One & $3(16.7)$ \\
\hline Two & $3(16.7)$ \\
\hline No & $12(66.7)$ \\
\hline \multicolumn{2}{|l|}{ Baseline use of oral corticosteroids } \\
\hline Yes & $2(11.1)$ \\
\hline No & $16(88.9)$ \\
\hline \multicolumn{2}{|l|}{ Baseline CDAI score } \\
\hline Mean \pm SD & $299.1 \pm 70.10$ \\
\hline Median $(95 \%$ CI $)$ & $288.8(264.2-334.0)$ \\
\hline \multicolumn{2}{|l|}{ Baseline SES-CD (centrally read) } \\
\hline Mean \pm SD & $14.8 \pm 10.04$ \\
\hline Median $(95 \% \mathrm{CI})$ & $10.0(9.6-20.2)$ \\
\hline
\end{tabular}

Data are presented as mean \pm standard deviation or $\mathrm{n}(\%)$ unless otherwise indicated

$C D$ Crohn's disease, $C D A I$ Crohn's Disease Activity Index, $C I$ confidence interval, ITT intent to treat, $S D$ standard deviation, $S E S-C D$ simple endoscopic score for Crohn's disease, $T N F$ tumor necrosis factor

\subsection{Change from Baseline in the Two-Item Patient-Reported Outcome Score}

At baseline, the median PRO2 score was 19.6 (95\% CI 16.8-22.3). For the ITT population, the median change from baseline in $\mathrm{PRO} 2$ score was -7.1 (95\% CI - 10.1 to -3.5$)$ at week $4(p=0.001),-8.7(95 \% \mathrm{CI}-11.1$ to $-6.0)$ at week $8(p<0.0001)$, and $-11.1(95 \% \mathrm{CI}-11.7$ to -5.2$)$ at week $12(p=0.0002)$. Mean changes from baseline in PRO2 score to each time point are shown in
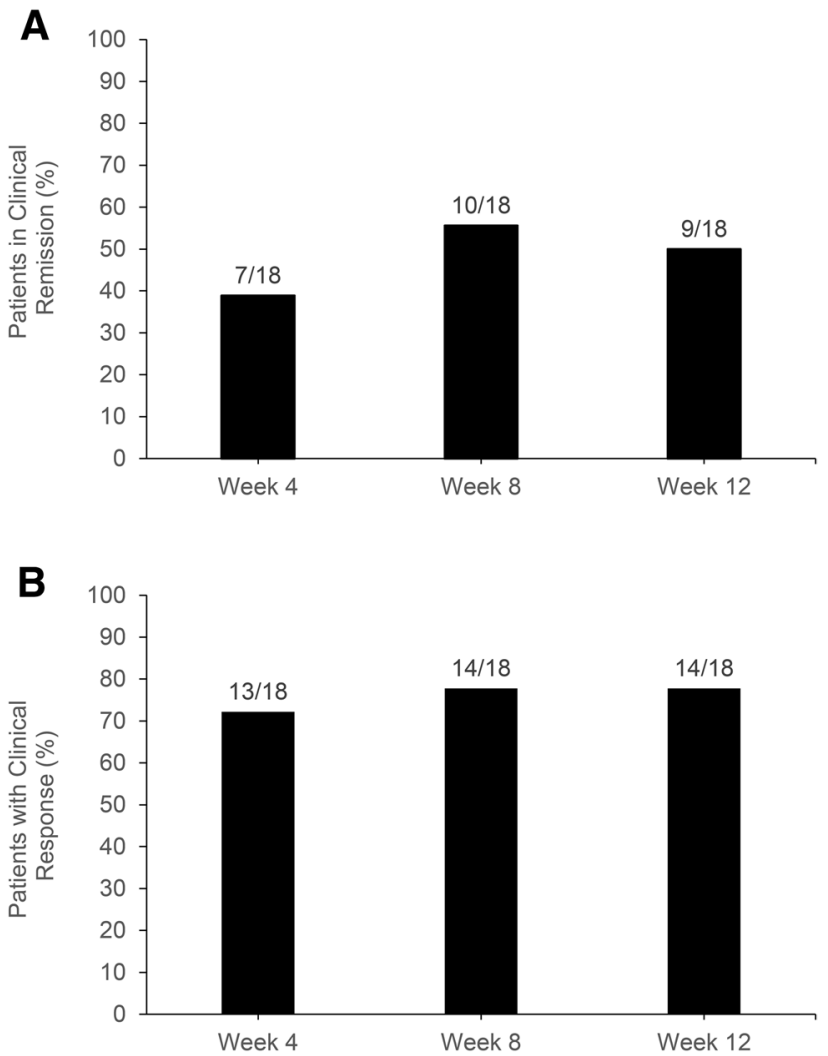

Fig. 1 Percentages of patients a experiencing clinical remission, defined as a Crohn's Disease Activity Index score of $<150$ at week 4, 8, and 12 following Mongersen treatment and $\mathbf{b}$ who had a clinical response, defined by a 100-point decrease in the Crohn's Disease Activity Index score at week 4, 8, and 12 following Mongersen treatment

Fig. 2b. Reductions were apparent in both the abdominal pain and the stool frequency subcomponents (data not shown).

\subsection{Change in Fecal Calprotectin, High-Sensitivity C-Reactive Protein, and Simple Endoscopic Score for Crohn's Disease}

The median fecal calprotectin level at baseline was 799.5 (95\% CI 720.6-1849.6). In the overall population, no clinically meaningful median changes from baseline in fecal calprotectin were observed during the 12-week treatment. Indeed, for the ITT population, the median change in fecal calprotectin level from baseline at week 4,8 , and 12 was 39.5 (95\% CI -498.4 to 56.6$),-72.5$ ( -555.9 to 809.7$)$, and -25 ( -362.8 to 979$)$, respectively. Next, we examined the median changes from baseline to week 12 in fecal calprotectin in the subgroup of patients with clinical response/ remission. Of 14 patients achieving clinical response/ remission, seven $(50 \%)$ exhibited a reduction in the fecal 
A
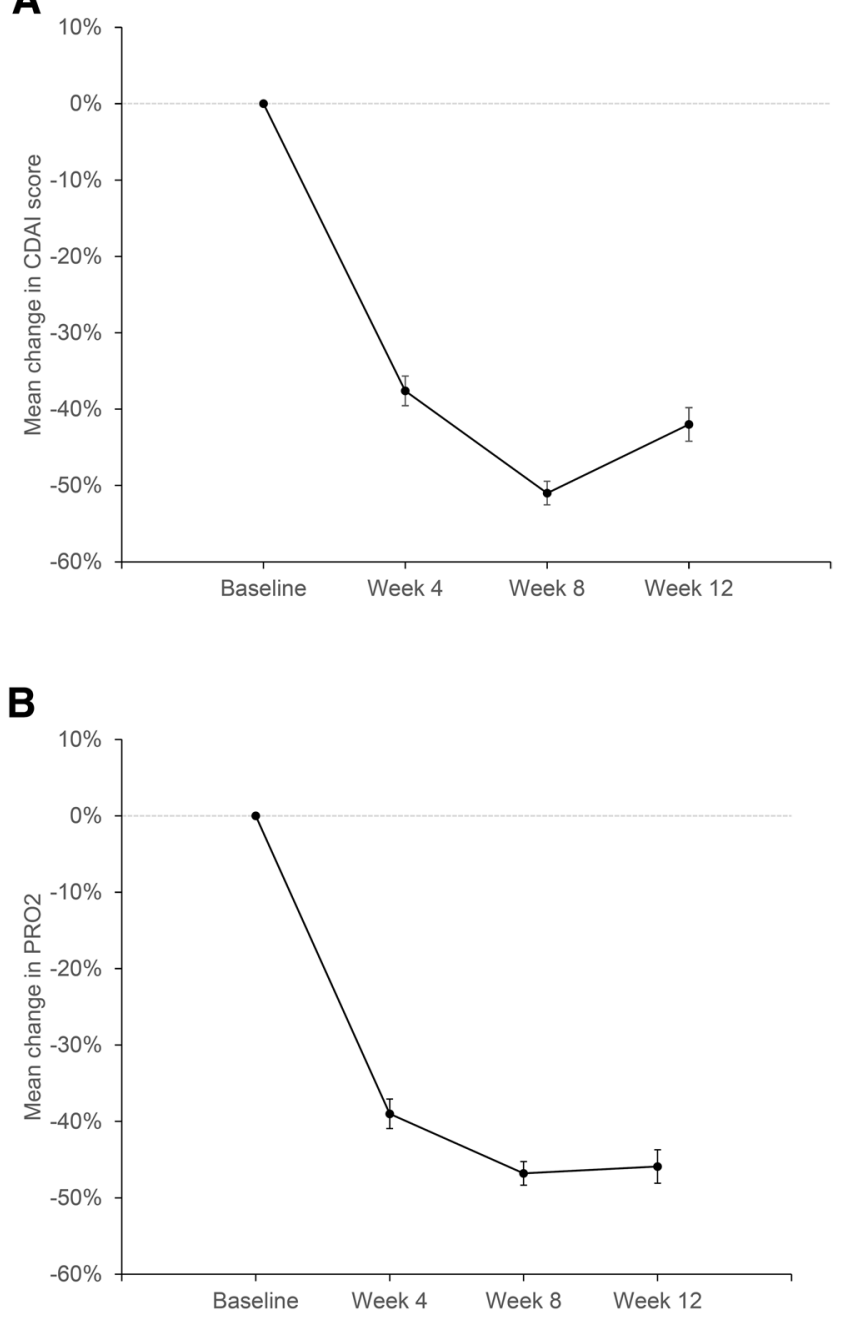

Fig. 2 Mean changes in a CDAI score from baseline at week 4, 8, and 12 and b PRO2 score from baseline at week 4, 8, and 12. Error bars show standard error of the mean. All 18 enrolled patients were included in the analysis (intention-to-treat population; last observation carried forward analysis). CDAI Crohn's Disease Activity Index, $P R O 2$ two-item patient-reported outcomes

calprotectin level from baseline (681 [145-3035]) to week 12 (393 [82-2231]) $(p=0.01)$. Of the total 18 patients, 11 (61\%) had baseline abnormal hsCRP (> $5 \mathrm{mg} / \mathrm{L}$ ); in nine of these patients, Mongersen treatment was associated with clinical remission/response, whereas the remaining two patients were unresponsive to the drug. In the subgroup of patients experiencing clinical remission/response, Mongersen induced a significant reduction in the median hsCRP value from baseline (28.4; range 6.5-118) to week 12 (11.3; range 3-66) $(p=0.039)$.

Overall, the mean \pm standard deviation baseline SES-CD (centrally read) score was $14.9 \pm 10.34$ and did not significantly change after 12 weeks of treatment $(15.5 \pm 11.4)$. Of the 17 patients for whom endoscopic assessment was available at week 12 , four (23.5\%) showed SES-CD $\geq 20 \%$ response.

\subsection{Safety Results}

All 18 enrolled patients were included in the safety analysis. During the treatment period, eight of 18 patients (44.4\%) reported at least one treatment-emergent adverse event (TEAE). The majority of TEAEs were reported in one patient each. The only TEAEs reported in more than one patient were pyrexia (four patients [22.2\%]) and arthralgia (two patients [11.1\%]). No patient died during the study, and no drug-related TEAEs, drug-related serious TEAEs, or TEAEs leading to GED0301 interruption were reported. One serious TEAE (anal abscess) was reported during the study in one patient. No treatment-emergent clinically meaningful abnormal hematology laboratory results, vital sign findings, or electrocardiogram values were reported during the study. No new safety signals were observed.

\subsection{Mongersen Reduces the Fraction of Circulating CCR9-Expressing CD45+ Cells}

The C-C chemokine receptor CCR9 is expressed on a certain subset of PBMC, and it mediates the homing to the intestinal mucosa of antigen-primed circulating mononuclear cells [22-24]. Peripheral blood samples were used to assess changes in the fraction of CCR9-expressing PBMC following Mongersen treatment. In particular, samples were collected at baseline and at week 12 from ten responders and two nonresponders. Only samples from patients enrolled at Tor Vergata Hospital were taken for flow-cytometric analysis. There was no significant change in the fraction of CD45+ cells and CD45+CD3+CCR9+ cells in the two nonresponders from baseline to week 12 (not shown). In contrast, in patients experiencing clinical remission/response, Mongersen induced a significant reduction in the fraction of both CD45+ cells and CD45+CD3+ cells expressing CCR9 (Fig. 3).

\subsection{In Vitro Knockdown of Smad7 with Different Batches of Mongersen}

Next, we investigated whether the different batches of Mongersen used in the various clinical trials inhibited Smad7 expression in cultured cells. For these studies, we used the human CRC cell line HCT-116, as these cells are easily transfected with Smad7 antisense oligonucleotides and represent a well-characterized model to monitor the functional consequence of Smad7 knockdown [25, 26]. The experiments included the negative control (lipofectamine); the reference batch NP004, which was used in both phase I and IGON1 phase II studies; the batch 
NP901, which was used in the present phase II study; and the batches NP425 and NP720, which were used in the phase III study. The batches NP004 and NP901 but not NP425 and NP720 downregulated Smad7 expression at both the RNA and the protein level, with NP004 being the most effective (Fig. 4a-c). As Smad7 plays a critical role in sustaining CRC cell proliferation [25, 26], we assessed the ability of the above mentioned batches to modulate HCT-116 cell growth. In line with the effects observed on Smad7 expression, only batches NP004 and NP901 significantly reduced the growth of HCT-116 cells (Fig. 4d).

\section{Discussion}

After the encouraging results of the phase I and II studies showing clinical and endoscopic benefits from Mongersen in active $\mathrm{CD}$, the phase III randomized double-blind, placebo-controlled multicentre study was terminated early by an independent committee because of a lack of both clinical and endoscopic efficacy $[12,17,19]$. In particular, in the latter study, only one-fifth of the patients receiving the highest dose of Mongersen (160 mg/day), which was associated with a $65 \%$ clinical remission rate in the phase II study, experienced a state of clinical remission, which was not significantly different from the placebo rate (25\%) [19].
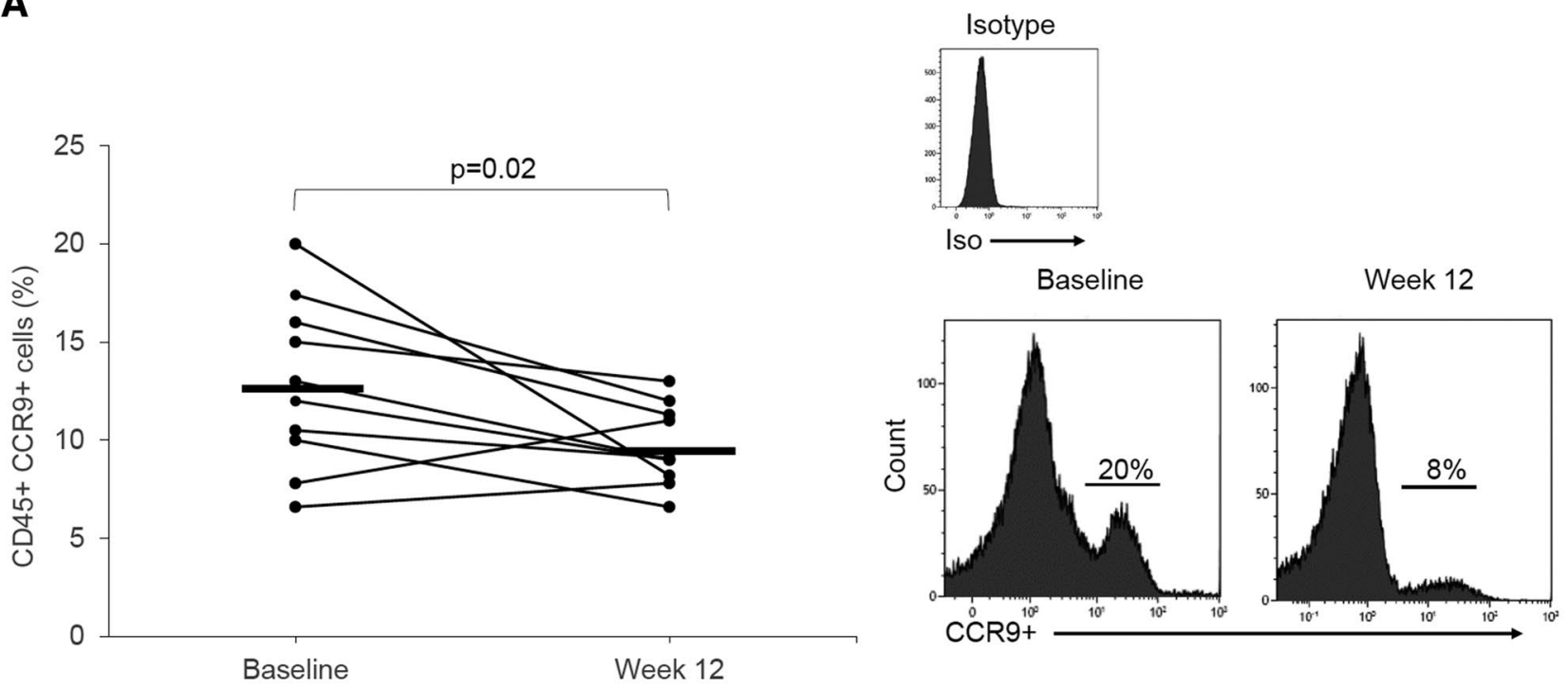

B
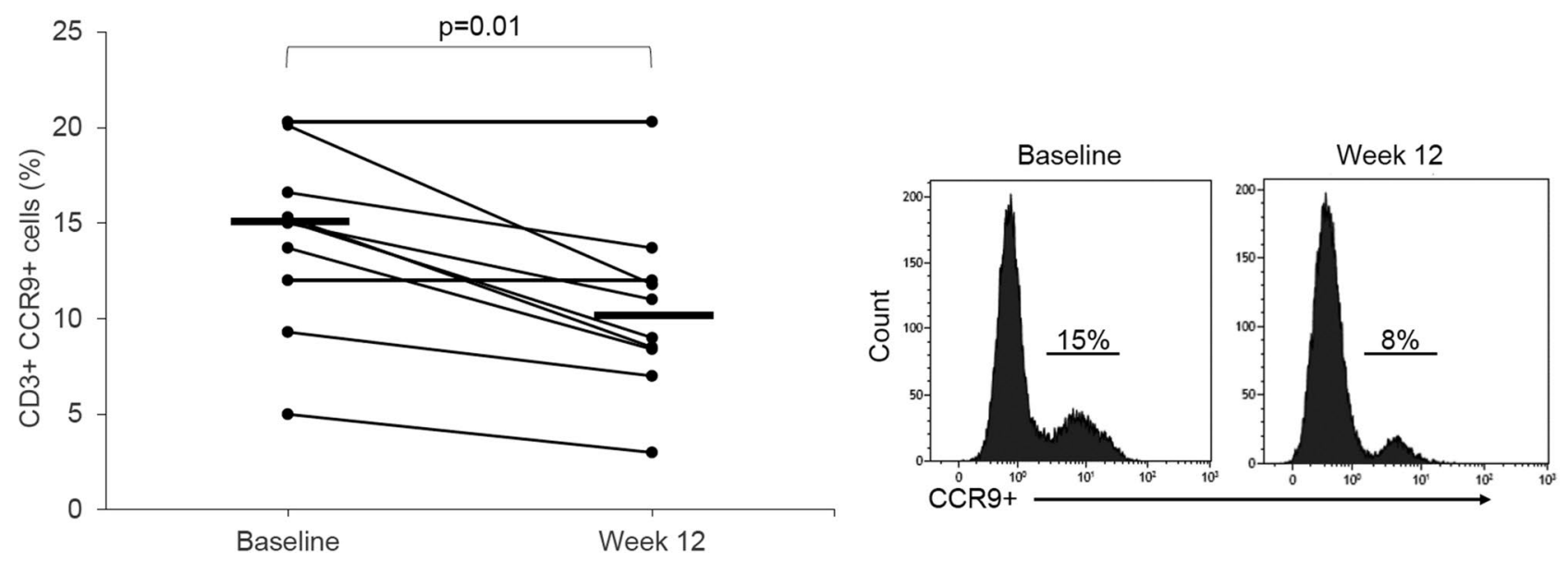

Fig. 3 Percentages of circulating CCR9-expressing a CD45+ cells and $\mathbf{b}$ CD45+CD3+ cells at baseline and at week 12 following Mongersen treatment in patients experiencing clinical remission/response at week 12. Each point in the graph indicates the fraction of positive cells in a single patient as evaluated by flow cytometry. Horizontal bars indicate the median values. Representative histograms are also shown for each subset of cells 
A

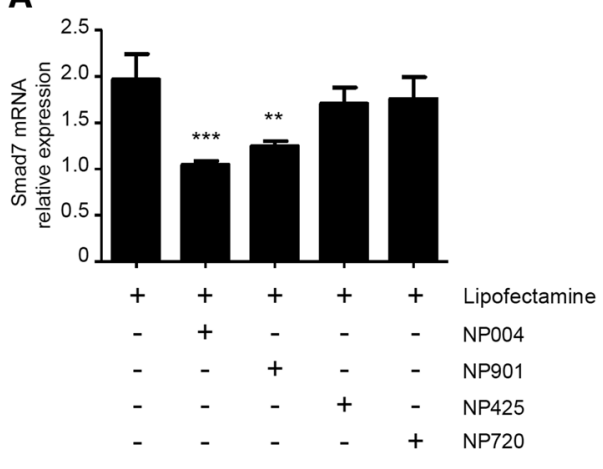

B

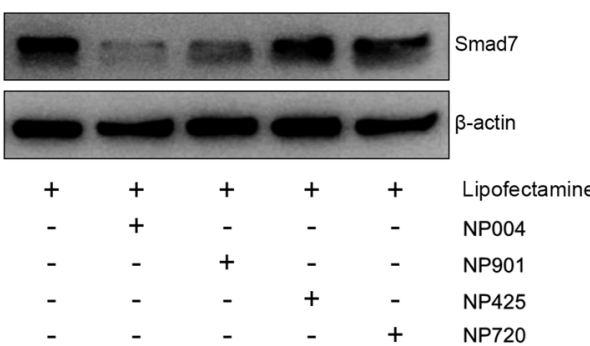

D

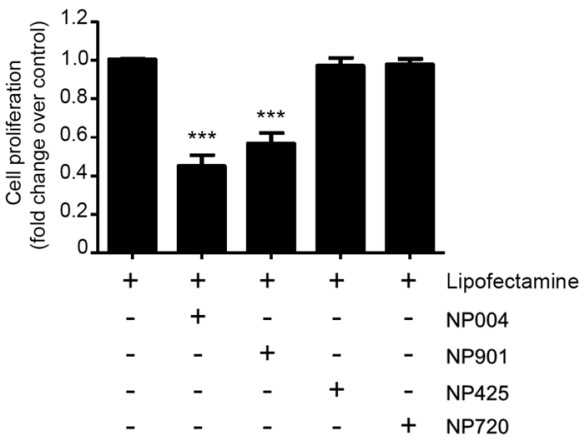

Fig. 4 In vitro effects of different batches of Mongersen on Smad7 expression and cell growth. a Effect of different batches of Mongersen on Smad7 mRNA expression. HCT-116 cells were transfected with either lipofectamine only (control) or the indicated batches of Mongersen as described in Sect. 2. Smad7 mRNA transcripts were evaluated using real-time polymerase chain reaction. Levels were normalized to $\beta$-actin. Values are mean \pm standard error of the mean of three experiments. NP901 vs. control, $* * P<0.01$; NP004 vs. control, $* * * P<0.001$. b Effect of different batches of Mongersen on Smad7 protein expression. HCT-116 cells were transfected with either lipofectamine only (control) or the indicated batches of Mongersen as described in Sect. 2. Whole-cell extracts were prepared and analyzed for Smad7 expression by western blotting. $\beta$-actin was used as a load-

The reasons for such a huge disparity between these two studies remain unknown.

We present the clinical outcomes of an open-label phase II study conducted at the same time as the phase III trial.
C

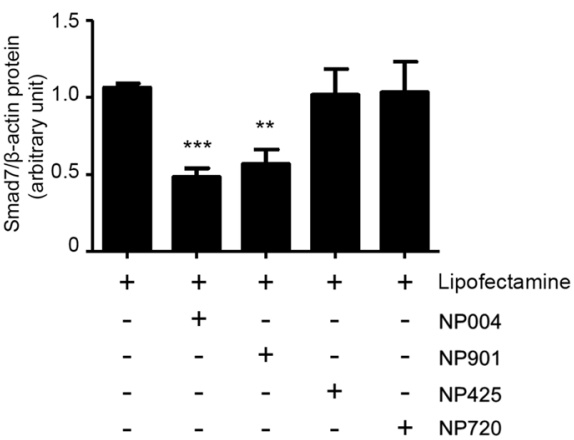

ing control. One of at least three representative experiments is shown. c Quantitative analysis of Smad7/ $\beta$-actin protein ratio in total extracts of HCT-116 cells transfected as indicated in (b), as measured by densitometry scanning of western blots. Values are expressed in arbitrary units and are the mean \pm standard error of the mean of three experiments. NP901 vs. control, $* * P<0.01$; NP004 vs. control, $* * * P<$ 0.001. d Effect of different batches of Mongersen on HCT-116 cell growth. Cells were transfected with either lipofectamine only (control) or the indicated batches of Mongersen and then labeled with carboxyfluorescein diacetate succinimidyl ester as described in Sect. 2. Cell proliferation was assessed by flow cytometry. NP004 or NP901 vs. control, $* * * P<0.001$

Moreover, we assessed the in vitro pharmacological activity of various batches of Mongersen used in the clinical trials. The study shows clinical improvements in patients with active $\mathrm{CD}$ following Mongersen treatment, thus confirming 
the benefits seen in the previous phase I and II studies. Improvements in both stool frequency and abdominal pain contributed to the reductions in CDAI and PRO2 scores. Since all patients enrolled for this study had active disease as defined by clinical, laboratory, and endoscopic scores, it is unlikely that the rates of clinical remission and clinical response seen in this study do not reflect the effect of Mongersen on the ongoing intestinal inflammation. Indeed, half of the patients who experienced clinical benefit exhibited reduced levels of fecal calprotectin, a biomarker of intestinal inflammation [27], following Mongersen treatment. Moreover, in the subgroup of patients with baseline hsCRP $>5 \mathrm{mg} / \mathrm{L}$ and experiencing clinical remission/response, Mongersen induced a significant reduction in the median hsCRP value from baseline to week 12. However, these findings indicate that, despite the clinical benefit, 12-week treatment with Mongersen is insufficient to suppress the mucosal inflammatory process. Indeed, evaluation of the effect of Mongersen on endoscopic outcomes revealed a SES-CD $\geq$ $20 \%$ response in only one-fifth of the patients. In this context, we would like to point out that our results are consistent with a recent phase II study investigating the effect of oral compounds (i.e., Janus kinase inhibitors) in active CD and showing significant clinical but not endoscopic improvements at week 12 [28].

Support for the modulating effect of the drug on the intestine-specific inflammatory responses comes from the demonstration that Mongersen significantly reduced the percentage of circulating mononuclear cells expressing CCR9, a chemokine receptor induced by TGF $\beta 1$ on leukocytes in the gastrointestinal-associated lymphoid tissue (e.g., Peyer's patches) and necessary for the homing of peripheral blood leukocytes to the intestinal mucosa [22-24].

The study confirmed the good safety profile of Mongersen $[12,17,19,21]$, and the safety findings documented were consistent with those expected in patients with active CD.

It is known that even small changes in the manufacturing processes of antisense oligonucleotides can cause significant differences in their clinical properties [29]. Consequently, batch-to-batch reproducibility of the active pharmaceutical ingredient is of utmost importance, as it can affect the downstream processing and hence the quality of the final product. Mongersen comprises 21 nucleotides bound by phosphorothioate linkages. Such phosphorothioate modifications are commonly introduced to enhance the protein-binding properties of the antisense oligonucleotide and consequently its pharmacological activity [30]. However, because the geometry of phosphorothioate installation cannot be controlled, all phosphorothioate-substituted antisense oligonucleotides comprise mixtures of drug diastereoisomers. Thus, patients treated with a specific phosphorothioate-modified antisense oligonucleotide likely receive a mixture of thousands of diastereoisomers that, in principle, may bear distinct three-dimensional structures and pharmaceutical properties [31,32].

Our in vitro data clearly indicated that various batches of Mongersen manufactured during the GED0301 program and used in the previous and current clinical trials differed in their ability to downregulate $\mathrm{Smad} 7$ expression, a function that is crucial in the mechanism of action of the drug. Specifically, batch NP901, which was used in the present clinical trial, reduced Smad7 expression in cultured HCT-116 cells. Downregulation of Smad7 in cells transfected with such a batch occurred at both the RNA and the protein level, confirming the antisense effect of the compound. Functionally, knockdown of Smad7 was followed by a significant reduction in HCT-116 cell proliferation, a finding that has been previously linked to the ability of Smad7 to regulate the activation of intracellular pathways that ultimately control colon cancer cell growth $[25,26]$. Interestingly, the inhibitory effect of such a batch on Smad7 was lower than that seen with the reference batch NP004, which was used in the IGON1 (phase II) study. In contrast, no downregulation of Smad7 RNA and protein expression was seen in HCT-116 cells transfected with two different batches of Mongersen, which were used in the phase III trial. Altogether, these data indicate that the ability of the various pharmaceutical batches developed during the whole GED0301 program to downregulate Smad7 in cultured cells differs.

As far as we know, in vitro cell culture studies are not considered as part of the quality process prior to the release of the batches used in phase III trials. In this regard, we strongly feel that, before setting up future trials employing Mongersen or other antisense oligonucleotide-based strategies, quality control studies aimed at determining batch purity and pharmacological activity are mandatory, as the high variability in the efficiency of different batches of the drug is not acceptable in the clinical use of such compounds.

Studies are now ongoing to ascertain whether and which physical/chemical changes that can occur during the largescale synthesis of the antisense oligonucleotides may have disrupted the pharmacological activity of some batches used in the phase III trial.

Limitations of this study include the small sample size and the lack of a placebo group. Furthermore, the design of the trial and selection of time points relied on evidence from previous phase I and II studies. Further work is needed to establish the optimal dose of the drug, duration of the treatment, and timing to assess the effect of Mongersen on mucosal/transmural lesions of the disease. Moreover, further studies are needed to ascertain whether the different in vitro activities of the batches of the drug can provide an explanation for the failure of the phase III study. 


\section{Conclusions}

The present findings support the clinical benefit of Mongersen in patients with active $\mathrm{CD}$ and show that various batches manufactured during the GED0301 program varied in their ability to inhibit Smad7 expression in cultured cells.

\section{Declarations}

Funding Open access funding provided by Università degli Studi di Roma Tor Vergata within the CRUI-CARE Agreement. The study was sponsored by Celgene and NograPharma (Mongersen's owner from April 2020).

Conflicts of Interest Giovanni Monteleone holds a patent for the use of Smad7 antisense oligonucleotides in CD. Irene Marafini, Carmine Stolfi, Edoardo Troncone, Elisabetta Lolli, Sara Onali, Omero Alessandro Paoluzi, Massimo C. Fantini, Livia Biancone, Emma Calabrese, Antonio Di Grazia, Ivan Monteleone, Marco Vincenzo Lenti, and Antonio Di Sabatino have no conflicts of interest that are directly relevant to the content of this article.

Ethics approval The study was conducted in accordance with the Declaration of Helsinki, the good clinical practice guidelines established by the International Conference on Harmonisation, and the applicable drug and data protection laws and regulations existing in Italy. Protocols, amendments, and informed consent documentation were reviewed and approved by the institutional review boards and independent ethics committee of each study center before the trial began.

Consent to participate Written informed consent was obtained from patients before they underwent screening for eligibility.

Consent for publication Not applicable.

Trial registration numbers NCT02685683, EudraCT 2015-001693-18

Availability of data and materials Data are available from the corresponding author upon reasonable request.

Code availability Not applicable.

Author contributions IMa wrote the paper and collected and analyzed data. CS performed experiments, wrote the paper, and collected and analyzed data. ET, EL, SO, OAP, MCF, LB, EC, and MVL recruited participants and oversaw the study. GM wrote the protocol, oversaw the study, recruited participants, wrote the paper, and analyzed data. ADG and IMo performed experiments. ADS recruited participants and oversaw the study. All authors approved the final manuscript as submitted and agree to be accountable for all aspects of the work.

Open Access This article is licensed under a Creative Commons Attribution-NonCommercial 4.0 International License, which permits any non-commercial use, sharing, adaptation, distribution and reproduction in any medium or format, as long as you give appropriate credit to the original author(s) and the source, provide a link to the Creative Commons licence, and indicate if changes were made. The images or other third party material in this article are included in the article's Creative Commons licence, unless indicated otherwise in a credit line to the material. If material is not included in the article's Creative Commons licence and your intended use is not permitted by statutory regulation or exceeds the permitted use, you will need to obtain permission directly from the copyright holder. To view a copy of this licence, visit http://creativecommons.org/licenses/by-nc/4.0/.

\section{References}

1. Abraham $\mathrm{C}$, Cho JH. Inflammatory bowel disease. $\mathrm{N}$ Engl $\mathrm{J}$ Med. 2009;361(21):2066-78. https://doi.org/10.1056/NEJMr a0804647.

2. Macdonald TT, Monteleone G. Immunity, inflammation, and allergy in the gut. Science. 2005;307(5717):1920-5. https://doi. org/10.1126/science.1106442.

3. Loftus EV Jr, Schoenfeld P, Sandborn WJ. The epidemiology and natural history of Crohn's disease in population-based patient cohorts from North America: a systematic review. Aliment Pharmacol Ther. 2002;16(1):51-60. https://doi.org/10. 1046/j.1365-2036.2002.01140.x.

4. Baumgart DC, Sandborn WJ. Crohn's disease. Lancet. 2012;380(9853):1590-605. https://doi.org/10.1016/S01406736(12)60026-9.

5. Digby-Bell JL, Atreya R, Monteleone G, Powell N. Interrogating host immunity to predict treatment response in inflammatory bowel disease. Nat Rev Gastroenterol Hepatol. 2020;17(1):920. https://doi.org/10.1038/s41575-019-0228-5.

6. Lichtenstein GR, Hanauer SB, Sandborn WJ, Practice Parameters Committee of American College of G. Management of Crohn's disease in adults. The American journal of gastroenterology. 2009;104(2):465-83; https://doi.org/10.1038/ajg.2008. 168 (quiz 4, 84)

7. Dignass A, Van Assche G, Lindsay JO, Lemann M, Soderholm $\mathrm{J}$, Colombel JF, et al. The second European evidence-based Consensus on the diagnosis and management of Crohn's disease: current management. J Crohns Colitis. 2010;4(1):28-62. https://doi.org/10.1016/j.crohns.2009.12.002.

8. Torres J, Bonovas S, Doherty G, Kucharzik T, Gisbert JP, Raine T, et al. ECCO guidelines on therapeutics in Crohn's disease: medical treatment. J Crohns Colitis. 2020;14(1):4-22. https:// doi.org/10.1093/ecco-jcc/jjz180.

9. Adamina M, Bonovas S, Raine T, Spinelli A, Warusavitarne J, Armuzzi A, et al. ECCO guidelines on therapeutics in Crohn's disease: surgical treatment. J Crohns Colitis. 2020;14(2):15568. https://doi.org/10.1093/ecco-jcc/jjz187.

10. Clark M, Colombel JF, Feagan BC, Fedorak RN, Hanauer SB, Kamm MA, et al. American gastroenterological association consensus development conference on the use of biologics in the treatment of inflammatory bowel disease, June 21-23, 2006. Gastroenterology. 2007;133(1):312-39. https://doi.org/ 10.1053/j.gastro.2007.05.006.

11. Monteleone G, Kumberova A, Croft NM, McKenzie C, Steer HW, MacDonald TT. Blocking Smad7 restores TGF-beta1 signaling in chronic inflammatory bowel disease. J Clin Investig. 2001;108(4):601-9. https://doi.org/10.1172/JCI12821.

12. Monteleone G, Fantini MC, Onali S, Zorzi F, Sancesario G, Bernardini $\mathrm{S}$, et al. Phase I clinical trial of Smad7 knockdown using antisense oligonucleotide in patients with active Crohn's disease. Mo Ther. 2012;20(4):870-6. https://doi.org/10.1038/mt.2011.290.

13. Troncone E, Marafini I, Stolfi C, Monteleone G. Transforming growth factor-beta1/Smad7 in intestinal immunity, inflammation, and cancer. Front Immunol. 2018;9:1407. https://doi.org/ 10.3389/fimmu.2018.01407.

14. Sedda S, Franze E, Bevivino G, Di Giovangiulio M, Rizzo A, Colantoni A, et al. Reciprocal regulation between $\operatorname{Smad} 7$ and Sirt1 in the gut. Front Immunol. 2018;9:1854. https://doi.org/ 10.3389/fimmu.2018.01854. 
15. Boirivant M, Pallone F, Di Giacinto C, Fina D, Monteleone I, Marinaro M, et al. Inhibition of Smad7 with a specific antisense oligonucleotide facilitates TGF-beta1-mediated suppression of colitis. Gastroenterology. 2006;131(6):1786-98. https://doi.org/ $10.1053 /$ j.gastro.2006.09.016.

16. Izzo R, Bevivino G, De Simone V, Sedda S, Monteleone I, Marafini I, et al. Knockdown of Smad7 with a specific antisense oligonucleotide attenuates colitis and colitis-driven colonic fibrosis in mice. Inflamm Bowel Dis. 2018;24(6):1213-24. https://doi. org/10.1093/ibd/izy062.

17. Monteleone G, Neurath MF, Ardizzone S, Di Sabatino A, Fantini MC, Castiglione F, et al. Mongersen, an oral SMAD7 antisense oligonucleotide, and Crohn's disease. N Engl J Med. 2015;372(12):1104-13. https://doi.org/10.1056/NEJMoa1407250.

18. Monteleone G, Di Sabatino A, Ardizzone S, Pallone F, Usiskin $\mathrm{K}$, Zhan X, et al. Impact of patient characteristics on the clinical efficacy of mongersen (GED-0301), an oral Smad7 antisense oligonucleotide, in active Crohn's disease. Aliment Pharmacol Ther. 2016;43(6):717-24. https://doi.org/10.1111/apt.13526.

19. Sands BE, Feagan BG, Sandborn WJ, Schreiber S, Peyrin-Biroulet L, Frederic Colombel J, et al. Mongersen (GED-0301) for active Crohn's disease: results of a phase 3 study. Am J Gastroenterol. 2020;115(5):738-45. https://doi.org/10.14309/ajg.0000000000 000493.

20. Bewtra M, Lichtenstein GR. Mongersen and SMAD-7 inhibition, not a lucky 7 for patients with IBD: when trial design is as important as disease therapy. Am J Gastroenterol. 2020;115(5):687-8. https://doi.org/10.14309/ajg.0000000000000564.

21. Feagan BG, Sands BE, Rossiter G, Li X, Usiskin K, Zhan X, et al. Effects of Mongersen (GED-0301) on endoscopic and clinical outcomes in patients with active Crohn's disease. Gastroenterology. 2018;154(1):61-64e6. https://doi.org/10.1053/j.gastro.2017. 08.035 .

22. Agace WW. T-cell recruitment to the intestinal mucosa. Trends Immunol. 2008;29(11):514-22. https://doi.org/10.1016/j.it.2008. 08.003 .

23. MacDonald TT, Monteleone G. IL-12 and Th1 immune responses in human Peyer's patches. Trends Immunol. 2001;22(5):244-7. https://doi.org/10.1016/s1471-4906(01)01892-0.

24. Papadakis KA, Prehn J, Moreno ST, Cheng L, Kouroumalis EA, Deem R, et al. CCR9-positive lymphocytes and thymus-expressed chemokine distinguish small bowel from colonic Crohn's disease.
Gastroenterology. 2001;121(2):246-54. https://doi.org/10.1053/ gast.2001.27154.

25. De Simone V, Bevivino G, Sedda S, Izzo R, Laudisi F, Dinallo V, et al. Smad7 knockdown activates protein kinase RNA-associated eIF2alpha pathway leading to colon cancer cell death. Cell Death Dis. 2017;8(3):e2681. https://doi.org/10.1038/cddis.2017.103.

26. Stolfi C, De Simone V, Colantoni A, Franze E, Ribichini E, Fantini $\mathrm{MC}$, et al. A functional role for Smad7 in sustaining colon cancer cell growth and survival. Cell Death Dis. 2014;5:e1073. https:// doi.org/10.1038/cddis.2014.49.

27. Tibble JA, Sigthorsson G, Bridger S, Fagerhol MK, Bjarnason I. Surrogate markers of intestinal inflammation are predictive of relapse in patients with inflammatory bowel disease. Gastroenterology. 2000;119(1):15-22. https://doi.org/10.1053/gast.2000. 8523.

28. Vermeire S, Schreiber S, Petryka R, Kuehbacher T, Hebuterne $\mathrm{X}$, Roblin X, et al. Clinical remission in patients with moderateto-severe Crohn's disease treated with filgotinib (the FITZROY study): results from a phase 2, double-blind, randomised, placebocontrolled trial. Lancet. 2017;389(10066):266-75. https://doi.org/ 10.1016/S0140-6736(16)32537-5.

29. Di Fusco D, Dinallo V, Marafini I, Figliuzzi MM, Romano B, Monteleone G. Antisense oligonucleotide: basic concepts and therapeutic application in inflammatory bowel disease. Front Pharmacol. 2019;10:305. https://doi.org/10.3389/fphar.2019. 00305 .

30. Koziolkiewicz M, Krakowiak A, Kwinkowski M, Boczkowska M, Stec WJ. Stereodifferentiation-the effect of P chirality of oligo(nucleoside phosphorothioates) on the activity of bacterial RNase H. Nucleic Acids Res. 1995;23(24):5000-5. https://doi. org/10.1093/nar/23.24.5000.

31. Iwamoto N, Butler DCD, Svrzikapa N, Mohapatra S, Zlatev I, Sah DWY, et al. Control of phosphorothioate stereochemistry substantially increases the efficacy of antisense oligonucleotides. Nat Biotechnol. 2017;35(9):845-51. https://doi.org/10.1038/nbt. 3948.

32. Knouse KW, deGruyter JN, Schmidt MA, Zheng B, Vantourout JC, Kingston C, et al. Unlocking P(V): reagents for chiral phosphorothioate synthesis. Science. 2018;361(6408):1234-8. https:// doi.org/10.1126/science.aau3369. 